\title{
MULTIPLIERS ON COMPLEMENTED BANACH ALGEBRAS
}

\author{
BOHDAN J. TOMIUK
}

(Communicated by Palle E. T. Jorgensen)

\begin{abstract}
Let $A$ be a semisimple right complemented Banach algebra, $L_{A}$ the left regular representation of $A$, and $M_{l}(A)$ the left multiplier algebra of $A$. In this paper we are concerned with $L_{A}$ and its relationship to $A$ and $M_{l}(A)$. We show that $L_{A}$ is an annihilator algebra and that it is a closed ideal of $M_{l}(A)$. Moreover, $L_{A}$ and $M_{l}(A)$ have the same socle. We also show that the left multiplier algebra of a minimal closed ideal of $A$ is topologically algebra isomorphic to $L(H)$, the algebra of bounded linear operators on a Hilbert space $H$. Conditions are given under which $L_{A}$ is right complemented.
\end{abstract}

\section{INTRODUCTION}

Let $A$ be a semisimple Banach algebra. In $\S 3$ we obtain some useful results for left (right) ideals in the algebras $A, L_{A}$, and $M_{l}(A)$. For example, we show that every closed left ideal $J$ of $A$ is a left ideal of $L_{A}$. Moreover, if $A$ contains a left approximate identity then $J$ is also a left ideal of $M_{l}(A)$. A semisimple annihilator right complemented Banach algebra has this property. Section 4 is devoted to the study of $L_{A}$, where $A$ is a semisimple right complemented Banach algebra. We show that $L_{A}$ is an annihilator algebra and that it is a closed ideal of $M_{l}(A)$. Each minimal closed ideal of $L_{A}$ is topologically algebra isomorphic to $\operatorname{LC}(H)$, the algebra of all compact linear operators on a Hilbert space $H$. Furthermore, $L_{A}$ is right complemented if and only if $x \in \operatorname{cl}_{L_{A}}\left(x L_{A}\right)$ for all $x \in L_{A}$. If $L_{A}$ is right complemented then it is a dual algebra.

\section{Preliminaries}

Let $A$ be a Banach algebra. For any subset $S$ of $A, l_{A}(S)$ and $r_{A}(S)$ will denote, respectively, the left and right annihilators of $S$ in $A$ and $\operatorname{cl}_{A}(S)$ will denote the closure of $S$ in $A$. The socle of $A$ will be denoted by $S_{A}$. By an ideal we will always mean a two-sided ideal unless otherwise specified. We call $A$ a modular annihilator algebra if every maximal modular left (right) ideal of $A$ has a nonzero right (left) annihilator. A semisimple Banach algebra with dense socle is modular annihilator [15, Lemma 3.11, p. 41]. We call $A$ an

Received by the editors October 20, 1990.

1991 Mathematics Subject Classification. Primary 46H10, 46H20; Secondary 46H15.

Key words and phrases. Right complemented Banach algebra, dual Banach algebra, left regular representation, left multiplier, abstract Segal algebra. 
annihilator algebra if for every closed right ideal $I, I \neq A, l_{A}(I) \neq(0)$, and for every closed left ideal $J, J \neq A, r_{A}(J) \neq(0)$. If, in addition, $r_{A}\left(l_{A}(I)\right)=I$ and $l_{A}\left(r_{A}(J)\right)=J$, then $A$ is called a dual algebra.

If $A$ is a semisimple Banach algebra and $K$ is an ideal of $A$, then $l_{A}(K)=$ $r_{A}(K)\left[15\right.$, p. 37]. We denote the common value $l_{A}(K)=r_{A}(K)$ by $K^{a}$. If $S_{A}^{a}=(0)$ then every nonzero left (right) ideal of $A$ contains a minimal idempotent ([3, p. 567] or [15, p. 34]).

Let $A$ be a semisimple Banach algebra. A linear mapping $T: A \rightarrow A$ is called a left multiplier if $T(x y)=T(x) y$ for all $x, y \in A$. Then $M_{l}(A)$ be the algebra of all left multipliers on $A$. Since every left multiplier on $A$ is continuous [6], $M_{l}(A)$ is a Banach algebra under the operator bound norm. For each $a \in A$, let $L_{a}$ be the operator on $A$ given by $L_{a}(x)=a x, x \in A$. Then $L_{a} \in M_{l}(A)$, for all $a \in A$, and the mapping $a \rightarrow L_{a}$ is a norm-decreasing algebra isomorphism of $A$ into $M_{l}(A)$ and embeds $A$ as a left ideal of $M_{l}(A)$, $[12,14]$. Let $L_{A}$ be the closure of $\left\{L_{a}: a \in A\right\}$ in $M_{l}(A)$. We call $L_{A}$ the left regular representation of $A$. In what follows we will identify $A$ as a left ideal of $M_{l}(A)$ and as a dense left ideal of $L_{A}$. In the terminology of [7], $A$ is an abstract Segal algebra in $L_{A}$. It is shown in [14] that every subalgebra $B$ of $M_{l}(A)$ such that $A \subset B$ is semisimple. Thus, in particular, $L_{A}$ is semisimple. (See also [12].)

All Banach algebras considered in this paper are over the complex field. When necessary we will denote the norm in a Banach algebra $A$ by $\|\cdot\|_{A}$. This will occur when two or more Banach algebras are involved at the same time. Otherwise the norm in $A$ will be denoted simply as $\|\cdot\|$.

Let $X$ be a Banach space. Then $L(X)$ will denote the algebra of all bounded linear operators on $X$ and $\mathrm{LC}(X)$ the subalgebra of all compact linear operators on $X$. If $S$ is a subspace of $X$ and $T \in L(X)$, then $T \mid S$ will denote the restriction of $T$ to $S$.

Let $A$ be a Banach algebra and let $L_{r}$ be the set of all closed right ideals in $A$. We say that $A$ is right complemented (r.c.) if there exists a mapping $p: I \rightarrow I^{p}$ of $L_{r}$ into itself (called a right complementor) having the following properties:

$\left(\mathrm{C}_{1}\right) \quad R \cap R^{p}=(0) \quad\left(R \in L_{r}\right)$;

$\left(\mathrm{C}_{2}\right) \quad R+R^{p}=A \quad\left(R \in L_{r}\right)$;

$\left.\left(\mathrm{C}_{3}\right) \quad\left(R^{p}\right)^{p}=R \quad(R \in L) r\right)$;

$\left(\mathrm{C}_{4}\right)$ if $R_{1} \subseteq R_{2}$ then $R_{2}^{p} \subseteq R_{1}^{p} \quad\left(R_{1}, R_{2} \in L_{r}\right)$.

A semisimple r.c. Banach algebra $A$ has dense socle [11, Lemma 5, p. 655] and, for every $x \in A, x \in \mathrm{cl}_{A}(x A)$ [1, Lemma 3, p. 39].

We put together several useful results in the following lemma.

Lemma 2.1. Let $A$ be a semisimple Banach algebra that is a dense subalgebra of a semisimple Banach algebra $B$. Then the following statements hold.

(i) If $S_{A}$ is a dense ideal of $B$ then $S_{A}=S_{B}$.

(ii) If $A$ is an annihilator algebra then $S_{A}$ is a dense ideal of $B$.

(iii) Assume that $S_{A}$ is dense in $A$. If $B$ is an annihilator algebra and $S_{A}$ is an ideal of $B$, then $A$ is an annihilator algebra.

Proof. (i) This is contained in the proof of [13, Lemma 4.1, p. 262].

(ii) This is proved in [14]. (See [14, Corollary 4.4, p. 128].) 
(iii) Suppose that $B$ is an annihilator algebra and that $S_{A}$ is an ideal of $B$. Then, by [4, Corollary, p. 1036], the identity embedding of $A$ into $B$ is continuous. Therefore $S_{A}$ is dense in $B$ and so $S_{A}=S_{B}$ by (i). Thus the norms $\|\cdot\|_{A}$ and $\|\cdot\|_{B}$ are equivalent on every minimal right ideal of $B$. Since $B$ is an annihilator algebra, [13, Corollary 6.11, p. 276] implies that every minimal right ideal of $B$ is a reflexive Banach space and every minimal idempotent of $B$ is full. The argument above shows that these properties also hold for minimal right ideals and minimal idempotents in $A$. Since $S_{A}$ is dense in $A$, we may now apply [13, Corollary 6.11, p. 276] to $A$ to show that $A$ is an annihilator algebra.

\section{IdEALS IN $A, L_{A}$, AND $M_{l}(A)$}

Proposition 3.1. Let $A$ be a semisimple Banach algebra and let $B$ be any subalgebra of $M_{l}(A)$ such that $A \subset B$. If $R$ is any nonzero right ideal of $B$ then $R \cap A \neq(0)$.

Proof. Suppose $R \cap A=(0)$. Since $A$ is a left ideal of $B$, we have $R A \subset R \cap A$ so that $R A=(0)$. Thus if $T \in R$ then $T L_{x}=0$ for all $x \in A$. Therefore $T L_{x}(y)=T(x y)=T(x) y=0$ for all $x, y \in A$, which shows that $T(x) \in$ $l_{A}(A)$ for all $x \in A$. The semisimplicity of $A$ implies that $T(x)=0$ for all $x \in A$. Thus $T=0$ and so $R=(0)$, a contradiction. Hence $R \cap A \neq(0)$.

Proposition 3.2. Let $A$ be a semisimple Banach algebra, and let $B$ be a subalgebra of $M_{l}(A)$ such that $A \subset B$. Then $S_{A}$ is a left ideal of $B$ and $S_{A} \subseteq S_{B}$. Proof. Let $e$ be a minimal idempotent of $A$. Since $A$ is a left ideal of $B$, $B e \subset A$ and therefore $B e=B e e \subset A e$. As $A e \subset B e$, we get $B e=A e$. Hence $S_{A} \subseteq S_{B}$.

In a similar way we can show that if $L_{A} \subset B$ then $S_{L_{A}} \subseteq S_{B}$.

Proposition 3.3. Let $A$ be a semisimple Banach algebra, and let $B$ be a subalgebra of $M_{l}(A)$ such that $A \subset B$. If $S_{A}^{a}=(0)$ then $S_{B}^{a}=(0)$ and every nonzero right ideal of $B$ contains a minimal idempotent of $A$.

Proof. Suppose that $S_{A}^{a}=(0)$. If $S_{B}^{a} \neq(0)$ then, by Proposition 3.1, $S_{B}^{a} \cap A \neq$ (0) and so contains a minimal idempotent $e$ of $A$. Since $S_{A} \subseteq S_{B}$, this means that $e \in S_{B}^{a} \cap S_{B}$ so that $e=e^{2}=0$, a contradiction. Therefore $S_{B}^{a}=(0)$.

Corollary 3.4. Let $A$ be a semisimple modular annihilator Banach algebra, and let $B$ be any subalgebra of $M_{l}(A)$ such that $A \subset B$. Then every nonzero right ideal of $B$ contains a minimal idempotent of $A$.

Proof. This is an immediate consequence of Proposition 3.3 since $S_{A}^{a}=(0)$ [3, Theorem 4.2, p. 269].

Proposition 3.5. Let $A$ be a semisimple Banach algebra. Then every closed left ideal of $A$ is a left ideal of $L_{A}$.

Proof. Let $J$ be a closed left ideal of $A$ and let $\mathbf{J}=\left\{L_{a}: A \in J\right\}$. We show that $\mathbf{J}$ is a left ideal of $L_{A}$. Let $T \in L_{A}$ and let $\left\{a_{n}\right\}$ be a sequence in $A$ such that $L_{a_{n}} \rightarrow T$. Let $y \in J$. Then $L_{a_{n}}(y)=a_{n} y \in J$ and $L_{a_{n}}(y) \rightarrow T(y)$. As $J$ is closed, $T(y) \in J$. But $T L_{y}(x)=T(y x)=T(y) x=L_{T(y)}(x)$, for all $x \in A$, implies that $T L_{y}=L_{T(y)}$. Hence $T L_{y} \in \mathbf{J}$ and so $\mathbf{J}$ is a left ideal of $L_{A}$. Identifying $J$ with $\mathbf{J}$ completes the proof. 
Corollary 3.6. Let $A$ be a semisimple Banach algebra. Then for every closed ideal $I$ of $A, \mathrm{cl}_{L_{A}}(I)$ is a closed ideal of $L_{A}$.

Proof. Let $\mathbf{I}=\left\{L_{a}: a \in I\right\}$. In view of Proposition 3.5 we need only show that $\operatorname{cl}_{L_{A}}(\mathbf{I})$ is a right ideal of $L_{A}$. Let $T \in \operatorname{cl}_{L_{A}}(\mathbf{I})$ and $S \in L_{A}$. Let $\left\{a_{n}\right\}$ be a sequence in $I$ such that $L_{a_{n}} \rightarrow T$ and $\left\{b_{n}\right\}$ be a sequence in $A$ such that $L_{b_{n}} \rightarrow$ $S$. Since $L_{a_{n} b_{n}} \in \mathbf{I}$ for all $n$ and $T S=\lim _{n \rightarrow \infty}\left(L_{a_{n}} L_{b_{n}}\right)=\lim _{n \rightarrow \infty} L_{a_{n} b_{n}}$, we see that $T S \in \mathrm{cl}_{L_{A}}(\mathbf{I})$. Identifying $I$ with $I$ completes the proof.

If $A$ has a left approximate identity (not necessarily bounded) then Proposition 3.5 takes the following more general form.

Proposition 3.7. Let $A$ be a semisimple Banach algebra with a left approximate identity. Then every closed left ideal of $A$ is a left ideal of $M_{l}(A)$.

Proof. Let $\left\{u_{\gamma}\right\}$ be a left approximate identity in $A$, and let $J$ be a closed left ideal of $A$. Let $a \in J$ and $T \in M_{l}(A)$. Since $\left\|a-u_{\gamma} a\right\| \rightarrow 0$ and $T$ is continuous, we have $\left\|T(a)-T\left(u_{\gamma} a\right)\right\| \rightarrow 0$. That is, $T\left(u_{\gamma} a\right)=T\left(u_{\gamma}\right) a \rightarrow T(a)$. Since $J$ is closed and $T\left(u_{\gamma}\right) a \in J$ for all $\gamma$, it follows that $T(a) \in J$. We can now apply the argument given in the proof of Proposition 3.5 to show that $J$ is a left ideal of $M_{l}(A)$.

Corollary 3.8. Let $A$ be a semisimple annihilator right complemented Banach algebra. Then every closed left ideal of $A$ is a left ideal of $M_{l}(A)$.

Proof. By [12, Theorem 3.7, p. 75], $A$ has a left approximate identity that is bounded in the norm of $L_{A}$. Application of Proposition 3.7 completes the proof.

\section{MAIN Results}

In this section we study $L_{A}$ where $A$ is a semisimple r.c. Banach algebra with a right complementor $p$. Since $A$ is semisimple, so is $L_{A}$.

Theorem 4.1. Let $A$ be a semisimple right complemented Banach algebra. Then $L_{A}$ is a semisimple annihilator algebra.

Proof. Let $K$ be a minimal closed ideal of $A$. Then $K$ is a topologically simple and semisimple r.c. Banach algebra [11, Lemma 1, p. 652]. Let $e$ be a minimal idempotent contained in $K$. Then $I=A e$ is a minimal left ideal of $K$ (and $A$ ) and so is a Hilbert space under an equivalent norm. If $I$ is finitedimensional this is clear, and if $I$ is infinite-dimensional this follows from [ 11 , Theorem 5, p. 652]. (See also [1, p. 40].) Denote this Hilbert space by $H$. Let $\varphi: a \rightarrow T_{a}$ be the representation of $K$ on $H$ corresponding to the left regular representation of $K$ on $I$, i.e., $T_{a}(x)=a x$ for all $x \in I$. Then $\varphi$ is a faithful, continuous, and strictly dense representation of $K$ on $H$. Hence if $K$ is finite-dimensional then $\varphi(K)=L(H)$. If $K$ is infinite-dimensional then it follows from [1, Theorem 1, p. 40] that $E T_{a} \in \varphi(K)$, for all orthogonal projections $E$ on $H$ and all $a \in K$. Thus, by [9, Theorem 1, p. 454], $\varphi(K)$ is a left ideal of $L(H)$. (See also [2, p. 391].) Since the socle of $\varphi(K)$ is dense in $\mathrm{LC}(H)$, the algebra of all compact linear operators on $H$, it follows that $\varphi(K)$ is a dense left ideal of $\operatorname{LC}(H)$. Thus $\varphi(K)$ is an abstract Segal algebra in $\mathrm{LC}(H)$ [ 7, Proposition 1.6, p. 299]. Therefore, by [12, Proposition 2.2, p. 73], the left regular representation $L_{K}$ of $K$ is topologically algebra isomorphic 
to $\mathrm{LC}(H)$. For each $a \in K$, let $L_{a}^{K}$ be the left multiplication by $a$ on $K$, i.e., $L_{a}^{K}(x)=a x$ for all $x \in K$. Then $L_{a}^{K} \in L_{K}$ and $L_{a}^{K}=L_{a} \mid K$. Since $A=K \oplus K^{p}$, there exists a constant $D_{K}>0$ such that if $x \in A$ and we write $x=x_{1}+x_{2}, x_{1} \in K$ and $x_{2} \in K^{p}$, then $\left\|x_{i}\right\| \leq D_{K}\|x\|$ for $i=1,2$.

For $T \in M_{l}(K)$, let $T^{\prime}$ be the mapping on $A$ given as follows: For $x \in A$, $x=x_{1}+x_{2}, x_{1} \in K$, and $x_{2} \in K^{p}$, define $T^{\prime}(x)=T\left(x_{1}\right)$. Then $T^{\prime}$ is linear and $\left\|T^{\prime}\right\| \leq D_{K}\|T\|$, where $\|T\|$ denotes the norm of $T$ over $K$. Clearly $\|T\| \leq\left\|T^{\prime}\right\|$. Moreover, using the fact that $K^{p}=l_{A}(K)=r_{A}(K)[11$, Lemma 1 , p. 652] and $K \oplus K^{p}=A$, it is easy to see that $T^{\prime} \in M_{l}(A)$. We have $T^{\prime} \mid K=T$ and $T^{\prime}\left(K^{p}\right)=(0)$. Also if $T_{1}, T_{2} \in M_{l}(K)$ then $\left(T_{1} T_{2}\right)^{\prime}=T_{1}^{\prime} T_{2}^{\prime}$. Hence the mapping $\rho_{K}: T \rightarrow T^{\prime}$ is a bicontinuous algebra isomorphism of $M_{l}(K)$ into $M_{l}(A)$ such that $\rho_{K}\left(L_{a}^{K}\right)=L_{a}$ for all $a \in K$. Thus, in particular, $\rho_{K}\left(L_{K}\right)$ is a closed subalgebra of $M_{l}(A)$. Since $L_{K}$ is the closure of $\left\{L_{a}^{K}: a \in K\right\}$ in $M_{l}(K)$, it follows that $\rho_{K}\left(L_{K}\right)$ is the closure of $\left\{L_{a}: a \in K\right\}$ in $M_{l}(A)$. Therefore $\rho_{K}\left(L_{K}\right) \subset L_{A}$ and $\rho_{K}\left(L_{K}\right)=\operatorname{cl}_{L_{A}}\left(\left\{L_{a}: a \in K\right\}\right)$. For convenience of notation, let $\mathbf{K}=\rho_{K}\left(L_{K}\right)$. Identifying $A$ as a subalgebra of $L_{A}$, we get $\mathbf{K}=$ $\mathrm{cl}_{L_{A}}(K)$. By Corollary $3.6, \mathbf{K}$ is a closed ideal of $L_{A}$. Since $\mathbf{K}$ is topologically algebra isomorphic of $\mathrm{LC}(H), \mathbf{K}$ is an annihilator algebra. Clearly $\mathbf{K}$ is a minimal closed ideal of $L_{A}$.

Let $\left\{K_{\alpha}: \alpha \in \Omega\right\}$ be the family of all distinct minimal closed ideals in $A$. By the argument above, for each $\alpha \in \Omega, \mathbf{K}_{\alpha}=\operatorname{cl}_{L_{A}}\left(K_{\alpha}\right)$ is a minimal closed ideal of $L_{A}$ and is an annihilator algebra. Since $\sum_{\alpha} K_{\alpha}$ is dense in $A$, it follows that $\sum_{\alpha} \mathbf{K}_{\alpha}$ is dense in $L_{A}$. Therefore, by [10, Theorem (2.8.29), p. 106], $L_{A}$ is an annihilator algebra.

Corollary 4.2. Let $A$ be a semisimple right complemented Banach algebra. Then the mapping $K \rightarrow \mathrm{cl}_{L_{A}}(K)$ is a one-to-one correspondence between the set of all minimal closed ideals of $A$ and the set of all minimal closed ideals of $L_{A}$, and $K=\operatorname{cl}_{L_{A}}(K) \cap A$. Moreover, every minimal closed ideal of $L_{A}$ is topologically algebra isomorphic to $\mathrm{LC}(H)$, the algebra of all compact linear operators on a Hilbert space $H$.

Proof. We only need to verify that $K=\operatorname{cl}_{L_{A}}(K) \cap A$, where $K$ is a minimal closed ideal of $A$, the rest is clear from the proof above. Let $\mathbf{K}=\mathrm{cl}_{L_{A}}(K)$ and let $K^{\prime}=\mathrm{K} \cap A$. Then $K^{\prime}$ is a closed ideal of $A$ and, therefore, is a semisimple right complemented Banach algebra in its own right [11, Lemma 1, p. 652]. Hence if $K \neq K^{\prime}$ then there exists a nonzero closed ideal $J$ in $K^{\prime}$ such that $K \oplus J=K^{\prime}$. Thus, in particular, $K J=(0)$. Since $\mathbf{K}=\mathrm{cl}_{L_{A}}(K)$, it follows that $\mathbf{K} J=(0)$. This is impossible since $J \subset \mathbf{K}$ and $\mathbf{K}$ is semisimple. Hence $J=(0)$ and so $K=\mathbf{K} \cap A$.

Corollary 4.3. A semisimple right complemented Banach algebra with a bounded right approximate identity is an annihilator algebra.

Proof. Since $A$ has a bounded right approximate identity, the norms $\|\cdot\|_{A}$ and $\|\cdot\|_{L_{A}}$ are equivalent on $A$. Hence the mapping $a \rightarrow L_{a}$ takes $A$ onto $L_{A}$.

Corollary 4.4. Let $A$ be a semisimple right complemented Banach algebra. Then $A$ is an annihilator algebra if and only if $S_{A}$ is an ideal of $L_{A}$. In this case we have $S_{A}=S_{L_{A}}$.

Proof. This follows immediately from Lemma 2.1 and Theorem 4.1. 
Theorem 4.5. Let $A$ be a semisimple right complemented Banach algebra and let $K$ be a minimal closed ideal of $A$. Then $M_{l}(K)$ is topologically algebra isomorphic to $L(H)$ for some Hilbert space $H$.

Proof. By the proof of Theorem 4.1, there exists a Hilbert space $H$ such that $K$ can be continuously embedded as a dense left ideal of $\mathrm{LC}(H)$ and $L_{K}$ is topologically algebra isomorphic to $\mathrm{LC}(H)$. Hence $M_{l}\left(L_{K}\right)$ is topologically algebra isomorphic to $M_{l}(\mathrm{LC}(H)$. In what follows we will identity $K$ as a dense left ideal of $\operatorname{LC}(H)$. Now, by [12, Proposition 3.1, p. 74], every $S \in M_{l}(K)$ has a unique extension $S^{\prime}$ to $L_{K}, S^{\prime} \in M_{l}\left(L_{K}\right)$, and $\left\|S^{\prime}\right\| \leq\|S\|$. Thus the mapping $S \rightarrow S^{\prime}$ is a continuous algebra isomorphism of $M_{l}(K)$ into $M_{l}\left(L_{K}\right)$. By [8, Lemma 2.1, p. 506], $M_{l}(\mathrm{LC}(H))$ is isometrically algebra isomorphic to $L(H)$. Therefore, $M_{l}\left(L_{K}\right)$ is topologically algebra isomorphic to $L(H)$ and so there exists a continuous algebra isomorphism $\sigma$ of $M_{l}(K)$ into $L(H)$. Since $K$ is a left ideal of $L(H)$, each $T \in L(H)$ gives rise to the left multiplier $S=L_{T} \mid K \in M_{l}(K)$. Hence $\sigma$ is onto and so $M_{l}(K)$ is topologically algebra isomorphic to $L(H)$. Thus the socle of $M_{l}(K)$ is mapped by $\sigma$ onto the socle of $L(H)$, and the socle of $L(H)$ is equal to the socle of $\operatorname{LC}(H)$. As $\operatorname{LC}(H)$ is topologically algebra isomorphic to $L_{K} \subset M_{l}(K)$, it follows that the socle of $M_{l}(K)$ is equal to the socle of $L_{K}$.

Corollary 4.6. Let $A$ be a semisimple right complemented Banach algebra and let $K$ be a minimal closed ideal of $A$. Then $M_{l}(K)$ is topologically algebra isomorphic to $M_{l}\left(L_{K}\right)$. Moreover, the socle of $M_{l}(K)$ is equal to the socle of $L_{K}$ so that, in particular, $L_{K}$ is a closed ideal of $M_{l}(K)$.

We will show below that also $L_{A}$ is an ideal of $M_{l}(A)$. We observe that if $I$ is a closed ideal of $A$, then $T(I) \subseteq I$ for all $T \in M_{l}(A)$. In fact, $I$ is a semisimple r.c. Banach algebra in its own right [11, Lemma 1, p. 652] and therefore has dense socle $S_{I}$. Since every minimal left ideal $J$ contained in $I$ is of the form $J=A e, e^{2}=e$, we get $T(J) \subseteq J$. Thus $T\left(S_{I}\right) \subseteq S_{I}$ and the continuity of $T$ implies that $T(I) \subseteq I$. Thus, in particular, $T \mid I \in M_{l}(I)$ for all $T \in M_{l}(A)$.

Let $K$ be a minimal closed ideal of $A$. Since $T \mid K \in M_{l}(K)$ for all $T \in$ $M_{l}(A)$, we see that $M_{K}=\left\{T \mid K: T \in M_{l}(A)\right\} \subseteq M_{l}(K)$. On the other hand, since for each $T \in M_{l}(K), T^{\prime}=\rho_{K}(T) \in M_{l}(A)$ and $T=T^{\prime} \mid K$ (see the proof of Theorem 4.1), we have $M_{l}(K) \subseteq M_{K}$. Hence $M_{K}=M_{l}(K)$. We know that $\rho_{K}\left(M_{l}(K)\right)$ is a closed subalgebra of $M_{l}(A)$. We claim that it is also an ideal of $M_{l}(A)$. Note that $\rho_{K}\left(M_{l}(K)\right)=\left\{T^{\prime}: T \in M_{l}(K)\right\}$. Let $T \in M_{l}(K), S \in M_{l}(A)$, and $x \in A$. Write $x=x_{1}+x_{2}$ with $x_{1} \in K$ and $x_{2} \in K^{p}$. Then $\left(T^{\prime} S\right)(x)=T^{\prime}\left(S\left(x_{1}+x_{2}\right)\right)=T^{\prime}\left(S\left(x_{1}\right)+S\left(x_{2}\right)\right)=T\left(S\left(x_{1}\right)\right)=$ $(T(S \mid K))\left(x_{1}\right)=(T(S \mid K))^{\prime}(x)$. Hence $T^{\prime} S=(T(S \mid K))^{\prime} \in \rho_{K}\left(M_{l}(K)\right)$. Likewise $S T^{\prime} \in \rho_{K}\left(M_{l}(K)\right)$. This verifies our claim.

Theorem 4.7. Let $A$ be a semisimple right complemented Banach algebra. Then $L_{A}$ is a closed ideal of $M_{l}(A)$.

Proof. To simplify notation, let $B=M_{l}(A)$. Let $A_{L}=\left\{L_{a}: a \in A\right\}$ and let $e$ be a minimal idempotent in $B$. By Corollary 3.4, $e B$ contains a minimal idempotent $f$ of $A_{L}$. We have $f=L_{g}$, for some minimal idempotent $g \in A$. Since $S_{L_{A}} \subseteq S_{B}$, it follows that $f$ is also a minimal idempotent of $B$ and $e B=f B$. Let $I=\mathrm{cl}_{B}(B e B)=\operatorname{cl}_{B}(B f B)$ and $K=\operatorname{cl}_{A}(A g A)$. Then $I$ (resp. 
$K)$ is a minimal closed ideal of $B$ (resp. $A$ ). Since $f \in \rho_{K}\left(M_{l}(K)\right) \cap I$, it follows that $\rho_{K}\left(M_{l}(K)\right) \cap I \neq(0)$ and therefore, by the minimality of $I$, $\rho_{K}\left(M_{l}(K)\right) \cap I=I$. This shows that $e \in \rho_{K}\left(M_{l}(K)\right)$. Now $L_{K} \subset M_{l}(K)$ and, by Corollary 4.6, $S_{L_{K}}=S_{M_{l}(K)}$. Hence $e \in \rho_{K}\left(L_{K}\right) \subset L_{A}$ and so $B e \subset S_{L_{A}}$. Thus $S_{B} \subseteq S_{L_{A}}$. As $S_{L_{A}} \subseteq S_{B}$, we obtain $S_{B}=S_{L_{A}}$. Since $S_{L_{A}}$ is dense in $L_{A}$ and $S_{L_{A}}=S_{B}$ is an ideal of $B$, it follows that $L_{A}$ is a closed ideal of $B$.

Corollary 4.8. Let $A$ be a semisimple right complemented Banach algebra. Then $S_{L_{A}}=S_{M_{l}(A)}$.

Corollary 4.9. Let $A$ be a semisimple right complemented Banach algebra. Then $A$ is an annihilator algebra if and only if $S_{A}=S_{M_{l}(A)}$.

Proof. This follows immediately from Corollaries 4.4 and 4.8.

Theorem 4.10. Let $A$ be a semisimple right complemented Banach algebra. Then $L_{A}$ is right complemented if and only if $x \in \mathrm{cl}_{L_{A}}\left(x L_{A}\right)$ for all $x \in L_{A}$.

Proof. To simplify notation, let $B=L_{A}$, and let $p$ be the right complementor on $A$. If $B$ is right complemented then, by [1, Lemma 3, p. 39], $x \in \operatorname{cl}_{B}(x B)$ for all $x \in B$; i.e., $B$ has approximate right units [7, p. 299]. Conversely suppose that $B$ has approximate right units. Let $L_{r}\left(\mathbf{L}_{r}\right)$ be the set of all closed right ideals in $A(B)$. Since $A$ also has approximate right units and $A$ is an abstract Segal algebra in $B$, by [7, Theorem 2.3, 299], the mapping $I \rightarrow \operatorname{cl}_{B}(I)$ is a bijection of $L_{r}$ onto $\mathbf{L}_{r}$ and $\operatorname{cl}_{B}(I) \cap A=I$. For $R \in \mathbf{L}_{r}$, let $R^{q}=\operatorname{cl}_{B}\left([R \cap A]^{p}\right)$. We claim that $q$ is a right complementor on $B$. To see that $\left(\mathrm{C}_{1}\right)$ is satisfied, let $I=R \cap R^{q}$. Then $I \cap A$ is a closed right ideal of $A$, $I \cap A \subset R \cap A$, and $I \cap A \subset R^{q} \cap A=[R \cap A]^{p}$. Hence $I \cap A \subset(R \cap A) \cap[R \cap A]^{p}=$ $(0)$. Therefore $I=(0)$. Property $\left(\mathrm{C}_{3}\right)$ also holds for $q$ since

$$
\begin{aligned}
\left(R^{q}\right)^{q} & =\operatorname{cl}_{B}\left(\left[\operatorname{cl}_{B}\left([R \cap A]^{p}\right) \cap A\right]^{p}\right)=\operatorname{cl}_{B}\left(\left([R \cap A]^{p}\right)^{p}\right) \\
& =\operatorname{cl}_{B}(R \cap A)=R .
\end{aligned}
$$

Moreover, if $R_{1}, R_{2} \in \mathbf{L}_{r}, R_{1} \subseteq R_{2}$, then $R_{2}^{q} \subseteq R_{1}^{q}$ since $R_{1} \cap A \subseteq R_{2} \cap A$ and $\left[R_{2} \cap A\right]^{p} \subseteq\left[R_{1} \cap A\right]^{p}$. Therefore $q$ satisfies $\left(\mathrm{C}_{4}\right)$. Since the norm $\|\cdot\|_{B}$ in $B$ has the property that $\|a\|_{B}=\sup \left\{\|a x\|_{A}:\|x\|_{A} \leq 1, x \in A\right\}$ for all $a \in A$, we can apply verbatim the argument in the proof of [13, Theorem 5.2(i), p. 265] to show that $q$ satisfies $\left(\mathrm{C}_{2}\right)$. Therefore $q$ is a right complementor on $B=L_{A}$ and this completes the proof.

Theorem 4.11. Let $A$ be a semisimple right complemented Banach algebra. If $L_{A}$ is right complemented then $L_{A}$ is a dual algebra.

Proof. Suppose that $L_{A}$ is right complemented. Then $x \in \operatorname{cl}_{L_{A}}\left(x L_{A}\right)$ for all $x \in L_{A}$. Since $L_{A}$ is an annihilator algebra, by [12, Theorem 3.6, p. 75], $L_{A}$ has a quasi-bounded left approximate identity so that $x \in \operatorname{cl}_{L_{A}}\left(L_{A} x\right)$ for all $x \in L_{A}$. Thus $x \in \operatorname{cl}_{L_{A}}\left(x L_{A}\right) \cap \operatorname{cl}_{L_{A}}\left(L_{A} x\right)$ for all $x \in L_{A}$. Therefore, by the proof of [10, Theorem (2.8.27), p. 104], $L_{A}$ is dual.

\section{REFERENCES}

1. F. E. Alexander, On complemented and annihilator algebras, Glasgow Math. J. 19 (1968), 38-45.

2. Representation theorems for complemented Banach algebras, Trans. Amer. Math. Soc. 148 (1970), 385-398. 
3. B. A. Barnes, Modular annihilator algebras, Canad. J. Math. 18 (1966), 566-578.

4. _ Some theorems concerning the continuity of algebra homomorphisms, Proc. Amer. Math. Soc. 18 (1967), 1035-1038.

5. S. B. Cleveland, Homomorphisms of non-commutative *-algebras, Pacific J. Math. 13 (1963), 1097-1109.

6. B. E. Johnson and A. M. Sinclair, Continuity of derivations and a problem of Kaplansky, Amer. J. Math. 90 (1968), 1067-1073.

7. M. Leinert, A contribution to Segal algebras, Manuscripta Math. 10 (1973), 297-306.

8. B. D. Malviya and B. J. Tomiuk, Multiplier operators on $B^{*}$-algebras, Proc. Amer. Math. Soc. 31 (1972), 505-510.

9. C. Pearcy and D. Topping, Sums of small numbers of idempotents, Michigan Math. J. 14 (1967), 453-465.

10. C. Rickart, General theory of Banach algebras, Van Nostrand, New York, 1960.

11. B. J. Tomiuk, Structure theory of complemented Banach algebras, Canad. J. Math. 14 (1962), 651-659.

12. __ Isomorphisms of multiplier algebras, Glasgow Math. J. 28 (1986), 73-77.

13. B. J. Tomiuk and B. Yood, Topological algebras with dense socle, J. Funct. Anal. 28 (1978), 254-277.

14. $\ldots$, Incomplete normed algebra norms on Banach algebras, Studia Math. 95 (1989), 119132.

15. B. Yood, Ideals in topological rings, Canad. J. Math. 16 (1964), 28-45.

Department of Mathematics, University of Ottawa, Ottawa, Ontario K1N 6N5, CanAda 